\title{
THE GENERALIZED $L_{p}$-WINTERNITZ PROBLEM
}

\author{
TONGYI MA
}

Abstract. This article introduced the notion of the $(i, j)$ type $L_{p}$-affine surface area of convex body in $\mathbb{R}^{n}$, and discussed its some proposition. In addition, we consider the more general $L_{p}$-Winterniz monotonicity problem about the $(i, 0)$ type $L_{p}$-affine surface area and $i$ th $L_{p}$ projection body in $\mathbb{R}^{n}$, and get a positive answer in all dimensions.

Mathematics subject classification (2010): 52A40, 52A20.

Keywords and phrases: Convex body, $i$ th $L_{p}$-curvature function, $i$ th $L_{p}$-curvature image, $(i, j)$ type $L_{p}$-affine surface area, $L_{p}$-Winterniz monotonicity problem.

\section{REFERENCES}

[1] W. BlaschKe, Vorlesungen über Differentialgeometric II, Springer-Verlag, Berlin, 1923.

[2] R. J. Gardner, Geometric Tomography, Cambridge Univ.Press, Cambridge, 1995.

[3] K. Leichtweiss, Affine Geometry of Convex Bodies, J.A. Barth, Heidelberg, 1998.

[4] E. Lutwak, Centroid bodies and dual mixed volumes, Proc. London Math. Soc. 60 (1990), 365-391.

[5] E. Lutwak, Extended affine surface area, Adv. Math. 85 (1991), 39-68.

[6] E. LuTWAK, The Brunn-Minkowski-Firey theory II: Affine and geominimal surface areas, Adv. Math. 118 (1996), 244-294.

[7] E. LutwaK, The Brunn-Minkowski-Firey theory I: mixed volumes and the Minkowski problem, J. Dffierential Geom. 38 (1993), 131-150.

[8] E. LutwaK, Dual mixed volumes, Pacific Journal of Mathematics 58, 2 (1975), 531-538.

[9] E. LutwaK, Mixed affine surface area, J. Math. Anal. Appl. 125 (1987), 351-360.

[10] S. J. LV AND G. S. LeNG, The $L_{p}$-curvature images of convex bodies and $L_{p}$-projection bodies, Proc. Indian Acad. Sci.(Math.Sci) 118, 3 (2008), 413-424.

[11] L. J. LiU, W. WANG AND B. W. HE, Fourier transform and $L_{p}$-mixed projection bodies, Bull. Korean Math. Soc. 47 (2010), 1011-1023.

[12] F. H. LU AND W. D. WANG, Inequalities for $L_{p}$-mixed curvature images, Acta Mathematica Scientia 30B (2010), 1044-102 (in Chinese).

[13] T. Y. MA AND W. D. WANG, On the analog of Shephard problem for the L $L_{p}$-projection body, Math. Inequal. Appl. 14, 1 (2011), 181-192.

[14] T. Y. MA, On $L_{p}$-mixed centroid bodies and dual $L_{p}$-mixed centroid bodies, Acta Mathematica Sinica, Chinese Series, 53, 2 (2010), 301-314 (in Chinese).

[15] T. Y. MA AND C. Y. LiU, The generalized Busemann-Petty problem for dual $L_{p}$-mixed centroid bodies, Journal of Southwest University, Natural Science Edition, 34, 4 (2012), 105-112 (in Chinese).

[16] T. Y. MA AND C. Y. LIU, The generalized Shephard problem for $L_{p}$-mixed projection bodies and Minkowski-Funk transforms, Journal of Shandong University (Natural Science) 47, 10 (2012), 21-30 (in Chinese).

[17] R. SchneIder, Convex Bodies: The Brunn-Minkowski Theory, Cambridge Univ. Press, Cambridge, 1993.

[18] C. M. PetTy, Geominimal surface area, Geom. Deom. Dedicata 3 (1974), 77-97.

[19] R. SChNeIDER, Zur einem Problem von Shephard uber die Projektionen konvexer Körper, Math.Z. 101 (1967), 71-82.

[20] W. D. Wang And G. S. Leng, $L_{p}$-mixed affine surface area, J. Math. Anal. Appl. 54 (2007), 1-14. 
[21] W. D. WAng AND G. S. Leng, $L_{p}$-dual mixed quermassintegrals, Indian J. Pure Appl. Math. 36, 4 (2005), 177-188.

[22] W. D. WAng And G. S. Leng, The Petty projection inequality for $L_{p}$-mixed projection bodies, Acta mathematica sinica, english series, 23, 8 (2007), 1485-1494 (in Chinese).

[23] J. YuAN, S. J. Lv AND G. S. LenG, The p-affine surface area, Math. Inequal. Appl. 10, 3 (2007), 693-702. 